\title{
AUTOMATED CONTROL SYSTEMS FOR ON-SITE CONCRETE DISTRIBUTION BY ROBOTIC MANIPULATORS
}

\author{
Prof. Dr.-Ing./Univ. Tokio Thomas Bock, Dipl.-Ing. Serguei Voltchkov \\ Technical University Munich \\ Chair for Building Realization and Informatics \\ Arcisstrasse 21, D-80333 Munich, Germany \\ Fon +498928922100 Fax +498928922102 \\ e-mail:svoltchkov@bri.arch.tu-muenchen.de
}

\begin{abstract}
Using boom-manipulators for raising high-altitude monolithic buildings with the help of sliding shutterings provides high economic indexes. Using boom-allocators in a combination with the detailed logistics at a building platform causes essential savings of time, that is one of the basic factors both in monolithic construction, and in economy generally. The significant effect brings application of microprocessor control tools of manipulating systems for on-site concrete distribution.
\end{abstract}

Keywords: robot, manipulator, automation, control, construction, concrete.

\section{ADVANTAGES OF APPLICATION OF ROBOTS - MANIPULATORS FOR CONSTRUCTION OF BUILDINGS FROM MONOLITHIC CONCRETE}

The technological process of construction of buildings from monolithic concrete and ferroconcrete consists of interdependent, interconnected procuring and building processes. The long-term experience of construction displays, that the wide application of pipeline transportation's technique is full coordinated to basic directions of intensification and complex mechanization of concrete operations, allowing to reduce the cost price of stacking during simultaneous increase of one worker's products more than twice, to lower complexity of construction of monolithic buildings, to increase productivity of work, to remove losses of concrete mixture at overloads, to improve conditions and labor safety, to increase quality and culture of manufacture.

The importance of this direction is determined by the factor, that nowdays is very actual problem of rational use of human resources in connection with absence of an opportunity of manufacture's expansion, using increasement of number of workers. For a realization of all aspects of vibrating is required the significant amount of hand-operated work, especially on rearrangement of vibrators. The vibration harmfully effects human organism. One of ways of enhancing of concrete mixtures' vibration by consolidation processes eliminating this effect, is the creation of the distance-controlled or automized mechanisms.

Thus, using of robots in construction promotes a heightening of work's productivity, improvement of quality of operational execution and reduction of danger for workers. The application of manipulators in monolithic construction allows to eliminate handoperated work and to automize processes of concrete mixture's stacking. Manipulators are especially perspective at concreting in sliding shuttering. In this case it is possible to create the robot-controlled sliding complexes ensuring automized installation of an armature, stacking of concrete mixture, its seal and control of shuttering's rise (Figure 1) [1]. They will enable considerably to expand volume of monolithic construction and to lower its cost price.

\section{OPERATIONAL ALGORIHMS}

The development of operational algorithms of the robot has a task of creating such movement rules, which would ensure purposeful movement of manipulators. Thus the scheduling of movement is executed by an operator in a way of motion with a gripper, and immediately program movement is carried out by either activation or deactivation of different drives of the robot-manipulator.

The control algorithm of the robot is based on its dynamic model. At the synthesis of the control of manipulators the nonlinearity and multiconnectivity of its dynamic model should be taken into account [2]. This allows to increase a system effectiveness of the control of robot. 


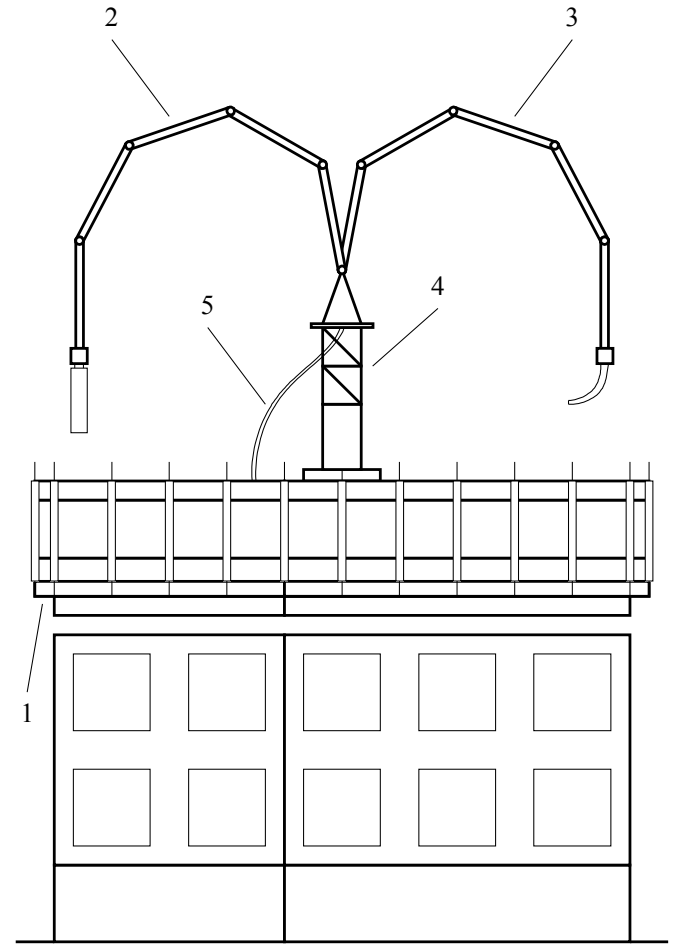

Figure 1. Robot system for concrete distribution and consolidation

1 - shuttering; 2 - manipulator with vibrator; 3 - manipulator with concrete pipeline; 4 - mast; 5 - concrete pipeline

Robot systems for concrete distribution and consolidation should have the large weight-lifting capacity (more than $100 \mathrm{kgs}$ ), that is why in this case the application of an electrohydraulic drive is expedient, as the pneumatic and electrical drives have not such large weight-lifting capacity or otherwise have considerably large overall dimensions.

The robot-manipulator for placing and consolidation of a concrete mixture is supposed to be used in a nondetermined medium with varying character of enclosing circumstances or during unregulated technological processes, if the strict programs are not able to guarantee a regularity and definition of robot's operation. In such case the most acceptable is to use an adaptive contour system of the program control ensuring the movement of an end-effector along the predetermined trajectory with established distribution of velocity values in time. It allows the robot to execute technological processes (transportation, placing and consolidation of concrete mixture), which are defining a continuity of the trajectory.

Our days programming of the robot is a prerogative of the operator controlling it. The method of programming by tutoring is the most prevailing. In this method the exemplary execution of some manipulations, which parameters are saved in a storing device of a control system, is carried out in a way of hand-operating control. The obtained this way rule of control represents a program trajectory of the robot's movement. This method of programming, despite of the simplicity, has a number of shortcuts, basic of which are temporal elimination of the robot out of technological process, impossibility of the account of dynamic links in a system, necessity of the highly skilled personnel's presence.

The method of an automatic programming of movements using computer is free of these shortcuts. Using this method the program trajectory is outputted immediately as a task to executive drives in real-time mode. The condition of a priori programming is possible also. In this case the program trajectory is brought beforehand in a buffer memory, from where it will be read with a necessary speed. The indicated modes eliminate presence of the human operator and, automizing process, allow to avoid the unjustified expenditures for "tutoring" of the robot.

\section{INFORMATION SYSTEM}

One of basic particulars of the robots, equipped by manipulation systems, is the ability of their active interaction with an environment on the basis of the information on properties and condition of an exterior medium, constructive elements of the robots and of the robot-manipulator as a whole. The most important functional tasks of information systems consist in:

- reception of the primary information as a particular physical signal;

- its transformation into a signal (it is possible of other physical nature), which format and style are adequate to a control system;

- processing the primary measured information and its representation in the format approaching for further use in a control system by the robotmanipulator for definition of character and parameters of its further movement.

Thus, the basic functionality of an information system consists in transformation of some physical characteristics to the information necessary to execute by the robot-manipulator specified technological operations. The information concerning condition of the robot itself and of the environment is represented on an output-port of sensors as signals, which values meet measured parameters.

The tasks executed by manipulation systems for placing and consolidation of a concrete mixture in shuttering forms [1] can be conditionally divided into some basic levels:

- orientation in working space and position on a starting point of concreting;

- movement of the manipulator (or manipulators) on the reference trajectory;

- avoidance of located on a shuttering's working floor obstacles;

- movement of the whole robot in "a real physical world" (movement of a mast according to the level of the working floor, transition to the following level after a work's completion on the current one). 
According to points, mentioned above, robotmanipulator for placing and consolidation of a concrete mixture in shuttering forms should have the information about

- geometrical sizes and configuration of shuttering;

- position of manipulators in space;

- dynamic loadings on links of manipulators;

- physical parameters of concrete mixture;

- weather conditions;

- conditions of vibration.

The information from sensors is transmitted to the computer, where with the help of specially designed for these purposes software has place its analysis, processing and output of signals to a control system of each manipulator separately and of the robot as a whole.

\section{CONTROL SYSTEM}

The control system is a major functional part of the robot-manipulator. In the modern robot it may have a complicated structure, consisting of several levels, providing transformation of tasks coming from the operator, information about properties of the exterior environment, program defining "behaviour" of the robot and, in particular, assignation of the rules of movement to its end-elements.

The first task of a control system consists in the definition of the rules of movement of the executive elements of a manipulation system providing required for movement execution of the end-effector's working process with the handled object. Essentially, this task is reduced to the analysis of positional functions. Other function of a control system consists in providing the execution of program movements, which are inputted. With this purpose are used both open, and closed-loop robot control systems. The movement control systems of executive elements are usually independent. Because of the effect of different disturbing factors on a system the real rules of movement of the executive elements differ from the programmed movements. There are errors, which will violate the working process. In order to widely decrease the number of errors in industrial robots, closed-loop control systems constructed on a principle of feedback will be used.

The dynamic characteristics of robot are described by nonlinear equations, and these are described by brightly expressed nonlinear links between separate levels of mobility apart from other factors. Position regulation is usually accomplished by standard (i.e. PI- or PID-) regulators. For the position regulation of the hydraulic cylinders of the drives a linear system model will be used, even though the drive has a nonlinear transfer function. However, the influence of nonlinearities to the dynamic properties of the controled object and, accordingly, to the regulation of a position is not taken into account. If the disturbing effects should be measured and univalent identified, their influence can be also taken into account. However it is completely clear, that the static error thus nevertheless exists. The advantage of such compensation of disturbing effects is the absence of necessity of application of an integral regulator and, as a consequence of it, the provision of good parameters for damping and speed of a control circuit. However the parameters of the controled object should be known rather precisely.

The robot control is calculated by the inverse model of the object, based on mathematical models, with known geometrical and mechanical parameters of the robot. This inverse model will be later used for movement registration of equations with a nonlinear character for the development of a control system. The exactitude of dynamic model plays in this case a very important role. A main problem for the regulation, based on a mathematical model, is the unreliability of the parameters of the object model.

The central algorithm controls drives of separate links of the manipulator, and also pressure of concrete pump or depth of immersing of the vibrator to concrete mixture (Figure 2). The compilance algorithm provides global algorithm with service datas and datas from the remote control unit. Thus it is possible both automatic operation of robot system, and its remote control through conductive communication lines or using radio, and also there is a present function of an emergency control of a system in case of emersion of a working platform unforeseen from the control algorithm situations. In the latter case driving robot system is carried out from the board of hand-operated control.

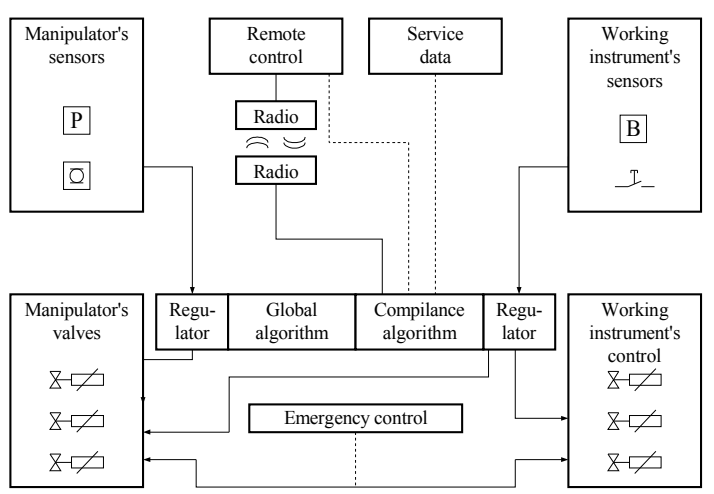

Figure 2. Architecture of manipulator control

Thus, the control process of each manipulator is carried out at two levels of hierarchy - with the help of the central or global and compilance control algorithms of manipulator with the working instrument. The highest level of robot system's hierarchy is the control algorithm by driving of both manipulators as unifide system (Figure 3 ). 


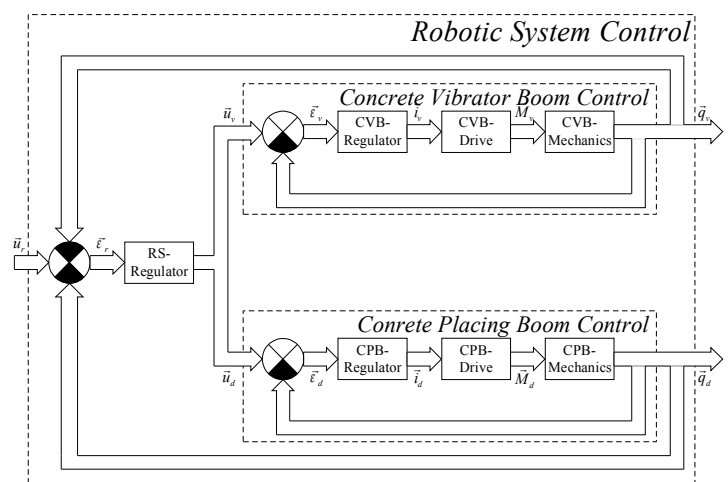

Figure 3. Structure of the robot's automatic control system

The control PC ensures reliable execution of a time schedule control with all hydraulic links both observance of given height and velocities of motion of the TCP. The sensors' signals act on actuators, and the high exactitude of positioning is reached with the help special transmitters of motion.

The central computer block calculates trajectories of driving links' manipulating system, stores them in memory (if necessary recurrings) and operates feeding of concrete to object. The radio control by process is supposed only in that case, when there are no signals or interferences, capable to cause violations in a radiocommunication on a building platform.

\section{REFERENCES}

[1] W. Sagorodnük, A. Bulgakov, S. Voltchkov. Robotersystem für das Belegen des Betons in Schalungsformen. 44. Internationales Wissenschaftliches Kolloquium 20.-23.09.1999. Band 2. Vortragsreihen. Mechatronik, Robotik und Automatisierungstechnik, Mikrosystemtechnik und Nanomaschinen, Innovative Werkstoffkonzepte. - c. 287-293.

[2] Ho-Gi Kim. Modellierung und Steuerung von Großmanipulatoren mit hydraulischen Antrieben. München, Wien: Hanser, 1994. 